\title{
Does Spine Posture Affect Isometric Torso Muscle Endurance Profiles in Adolescent Children?
}

\author{
Aleksandar Dejanovic $^{1}$, Edward D. J. Cambridge ${ }^{2}$, Stuart McGill $^{2 *}$ \\ ${ }^{1}$ Vertex-Human Performance Lab, Novi Sad, Serbia \\ ${ }^{2}$ Department of Kinesiology, University of Waterloo, Waterloo, Canada \\ Email: ${ }^{*}$ mcgill@uwaterloo.ca
}

Received March $8^{\text {th }}, 2013$; revised April $8^{\text {th }}, 2013$; accepted April 15 ${ }^{\text {th }}, 2013$

\begin{abstract}
Copyright (C) 2013 Aleksandar Dejanovic et al. This is an open access article distributed under the Creative Commons Attribution License, which permits unrestricted use, distribution, and reproduction in any medium, provided the original work is properly cited.
\end{abstract}

\begin{abstract}
The purpose of this cross-sectional study was to examine mean values of isometric torso muscle profiles of four spinal postures (good posture, thoracic kyphosis, lumbar lordosis and scoliosis) among 743 children from the ages of 7 to 14 years old. It was hypothesized that having good posture, thoracic hyper-kyphosis, lumbar hyper-lordosis and scoliosis is linked to different isometric torso muscle endurance profiles. Torso muscle endurance, established through four tests (Biering-Sørensen Test for extensor endurance, Flexor Endurance Test and right and left Side Bridge Tests for lateral endurance) performed in random order and spine postural screening categorized subjectively by observation was measured. Posture was proved to be linked to endurance scores. Hyper-lordotic spines demonstrated a decreased endurance compared to the three other postures $(\mathrm{F}=5.344 ; p<0.01)$; pairwise comparisons confirmed these differences $(p<0.05)$. Trends further suggested that hyper-lordosis was detrimental in lateral chain torso endurance while a hyper-kyphotic spine was more resilient in anterior chain torso endurance. Understanding the relationship between posture and endurance may be beneficial in clinical, as well as coaching/teaching settings.
\end{abstract}

Keywords: Isometric Torso Muscle Endurance Tests; Children; Spine; Posture

\section{Introduction}

Links between standing posture, chronic muscle contraction, extensor muscle endurance and back pain motivated this study. Spinal posture is a highly complex system consisting of the bony architecture and passive ligaments, the active musculature, and the neurological control system. For children, in particular, spine posture appears to be a very critical part of the developing neuromuscular system. For example, prolonged sitting could be a concern given in the classroom environment; posterior pelvis rotation has been identified as a risk factor for the development of subsequent back pain (Harrison et al., 1999). These sitting postures reduce lumbar lordosis, and increase both muscular tension and intervertebral disc pressure (Harrison et al., Wilke et al., 1999). These loads are coupled with growth spurts between 9.5 and 12.5 years of age (Malina et al., 2004). It has been demonstrated that improper spinal loading can influence muscle strength and motor performance (Malina et al., 2004), change muscle length (Kendall et al., 2005), alter torso muscular endurance (Dejanovic et al., 2012), influence spinal posture (Hrysomallis \& Goodman, 2001) and cause an increase in spinal curvature (Escalada et al., 2005; Sanders et al., 2006). Not only does improper functioning appear to change spine posture, but changes in the size of anteroposterior spinal curvatures may derange functioning of the trunk muscles (Anwajler et al., 2006). Furthermore, longer abdominal and shorter erector spinae muscles were linked with a hyper-lordotic lumbar curvature

* Corresponding author.
(Toppenberg \& Bullock, 1986). Despite the apparent links between posture and growth of children, and between muscle function and the risk for the development of LBP, little investigation has been conducted in this area.

Normal healthy posture can be described as a state of balance between muscular and bony structures, which minimizes local passive tissue stress and strain (Griegel-Morris et al., 1992). Deviated spine posture may lead to a range of disorders (Breen et al., 2007), including: low back pain (Harrison et al., 2002; McGill, 1997). However, improper posture may also occur as a result of fatigue, which may increase the risk of injury (Sparto et al., 1997). McGill (2007) demonstrated that poor standing posture can result in muscle pain from chronic muscle contraction of the erector spinae group. Increased segmental or overall lumbar lordosis may be associated with LBP (Dankaerts et al., 2006).

While sagittal plane posture appears important, they are also frontal plane deformities. For example, adolescent idiopathic scoliosis is a spinal deformity commonly being noticed between 10 and 16 years of age (Reamy \& Slakey, 2001) especially in young girls (Weinstein, 1994). Scoliosis can be associated with back pain (Ramirez et al., 1997), alter balance control (Gauchard et al., 2001), and different muscle activation strategies (Stokes et al., 2004).

Little is known about the variability of children's standing posture (McEvoy \& Grimmer, 2005). Given the rationale above, the aim of this cross-sectional study was to establish normative data for adolescent children for four spine postures (normal, 
thoracic hyper-kyphosis, lumbar hyper-lordosis and scoliosis) and whether there was a link to isometric torso muscle endurance. Identifying standing postural types and their torso muscle endurance profiles may provide insight into the development of low back pain in school children, together with guiding a therapeutic approach for preventing and managing improper spine posture and pain.

\section{Methods}

Spine posture and torso muscular endurance were evaluated to establish a database, and to evaluate the relationship between posture and endurance among children. The testing, data collection, methods and posture screening were presented to and approved by the Dean and Parents' Committee of the Elementary School, City of Novi Sad, Republic of Serbia, Medical Ethics Committee of Institute for Health Protection and Prevention of Children and Youth of Vojvodina, City of Novi Sad, Republic of Serbia. Informed consent forms were signed by all subjects and their parents prior to data collection. All children and parents were familiarized with the posture screening and each test protocol. The inclusion criteria for participants were: 1) aged from 7 to 14 years of age, 2) no neurological injury of the hips, lower back or cervical spine, 3) no numbness, weakness or injury in the upper or lower extremities, 4) no spinal structural deformities, 5) no current signs or symptoms of headache, 6) all subjects were healthy for the 4 weeks prior to testing.

\section{Subjects}

This study involved 753 children aged 7 to 14 from one Serbian elementary school, 394 boys and 359 girls. The children were assessed and grouped into 4 posture groups. For the boys' subset, per posture group numbers were: good posture $(n=49)$, lordosis $(\mathrm{n}=112)$, kyphosis $(\mathrm{n}=138)$ and scoliosis $(\mathrm{n}=95)$. In the girls' subset, per posture group numbers were: good posture $(\mathrm{n}=79)$, lordosis $(\mathrm{n}=123)$, kyphosis $(\mathrm{n}=75)$ and scoliosis $(\mathrm{n}$ $=82$ ). The distribution of cases by posture included subjects' classification by gender and by age (Table 1). The "young" group was comprised of those between the ages of 7 and 10 years, while the "old" group was of those between 11 and 14 years.

\section{Postural Assessment}

Boys and girls were separated for postural screening purposes and were observed separately. Children conformed to the dress code agreed in the informed consent. Boys were dressed only in underwear while girls were in elastic polyester or cotton under crops and sport top bra.

Posture was assessed subjectively in the sagittal and frontal planes using the protocols of Mulhearn and George (1999) and Kendall et al. (2005). Children stood quietly with their arms relaxed by their sides. All subjects were assessed by the same Kinesiotherapist with 15 years of clinical experience in orthopedic physical therapy and kinesiotherapy. Instead of a side plumb line used by Mulhearn and George (1999), a posture cage with $5 \times 5 \mathrm{~cm}$ net (see Figure 1) covered the torso. In the sagittal plane, the cage net was positioned $1 \mathrm{~cm}$ away from subject's body, between the subject and the observer with the plumb line passing through the mastoid process. In the frontal plane the line passed through the middle of the body (Kendall et al., 2005). Postures were classified as - thoracic kyphotic (swayback), lumbar lordotic and good posture. The fourth posture (scoliotic) was determined with Adam's forward bend, test since it is more sensitive than the scoliometer (Côté et al., 1998). The Adam's test began in a standing position, the subject was then asked to bend forward looking down, keeping the feet approximately $10-15 \mathrm{~cm}$ apart, knees fully extended, shoulders loose and hands positioned in front of thighs and knees, with elbows straight and palms opposed. Leg length discrepancy was not assessed.

\section{Endurance Tests}

Four tests were used to establish isometric torso muscle endurance, according to McGill et al. (1999): back extension test, right and left side bridge torso test and flexor endurance test.

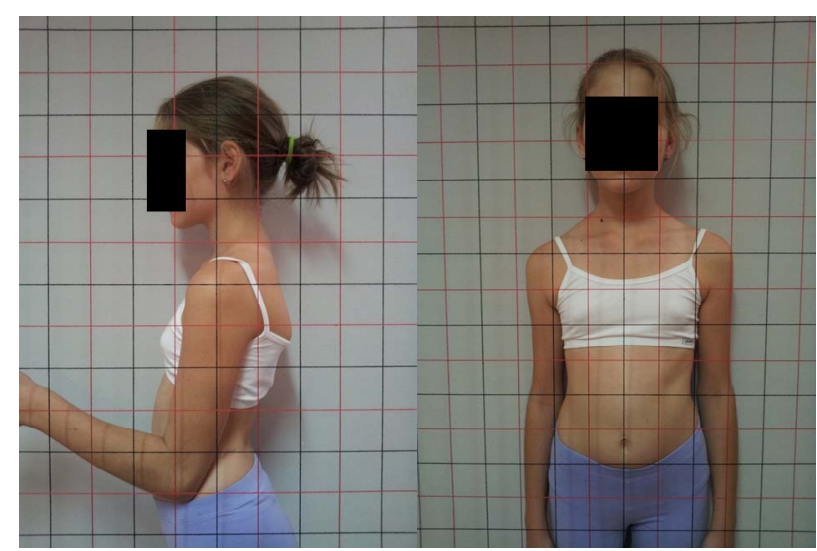

Figure 1.

Postural assessment with cage net in frontal and sagittal plane (examples).

Table 1.

Distribution of cases by postural categorization for all subjects $(n=753)$ and by gender and age group.

\begin{tabular}{cccccc}
\hline \multirow{2}{*}{ Posture } & \multirow{2}{*}{ All Cases } & \multicolumn{2}{c}{ Gender } & \multicolumn{2}{c}{ Age Groups } \\
\cline { 3 - 6 } & & male & female & Young & Old \\
\hline Good & $128(17 \%)$ & $49(12 \%)$ & $79(22 \%)$ & $66(20 \%)$ & $62(15 \%)$ \\
Lordotic & $235(31 \%)$ & $112(28 \%)$ & $123(34 \%)$ & $136(41 \%)$ & $99(24 \%)$ \\
Kyphotic & $213(28 \%)$ & $138(35 \%)$ & $75(20 \%)$ & $66(20 \%)$ & $147(35 \%)$ \\
Scoliotic & $177(24 \%)$ & $95(24 \%)$ & $82(23)$ & $68(20 \%)$ & $109(26 \%)$ \\
Total & $753(100 \%)$ & $394(100 \%)$ & $359(100 \%)$ & $336(100 \%)$ & $417(100 \%)$ \\
\hline
\end{tabular}


McGill et al. (1999) documented that these tests have high reliability coefficient $>0.97$ when tested consecutively over a five-day period, while Evans et al. (2007) showed high reliability for lateral endurance tests.

Back extension test (BET):

Back muscle endurance followed the original Biering-Sørensen position. Subjects layed on a Swedish Box $(1500 \times 1100 \times$ $500 \mathrm{~mm}$ ) covered with a soft pad (50 mm thick), elevated 500 $\mathrm{mm}$ from the floor, with a soft pad for arm support placed on the floor. Straps secured the legs and were lined with soft pads to prevent discomfort. Subjects lay prone over the end of the bench with fixed legs by three seat belts or with the ankles held by another person. Upon request, subjects crossed their arms over the chest and maintained the torso parallel with the ground. During the test, the subjects were allowed to be verbally corrected twice to maintain the regular position before the test stopped (see Figure 2).

Side bridge torso test ( $L S B \& R S B)$ :

In the side bridge test, the same mat $(2000 \times 1000 \times 50 \mathrm{~mm})$ was used, soft enough to prevent discomfort in the elbows, knees and feet. For this test, the subject lay on their side on their forearm, with the elbow flexed to $90^{\circ}$, holding their torso off the floor. Legs were extended; the top foot was placed in the front of the lower foot for support (see Figure 3).

Flexor Endurance Test (FET):

The flexor endurance test was conducted on a Judo mat $(2000 \times 1000 \times 50 \mathrm{~mm})$ with a wooden box jig angled $50^{\circ}$ from the floor (after McGill et al., 1999), and toes were covered with soft pads and secured with toe straps. Subjects began in a sit-up position, crossed arms over the chest with hands placed on the opposite shoulder, toes secured under toe straps or supporters palms, and the back resting against the jig angled $50^{\circ}$ from the floor. Knees and hips were flexed to $90^{\circ}$. Upon request the jig was pulled back $10 \mathrm{~cm}$ while the subject held their position as long as possible (see Figure 4).

Time was measured with a TAG Heuer electronic Microsplit MS200 stopwatch. During each test, two assistants were present beside the subject for safety and injury prevention reasons. Tests ended if subject could not maintain straight torso position

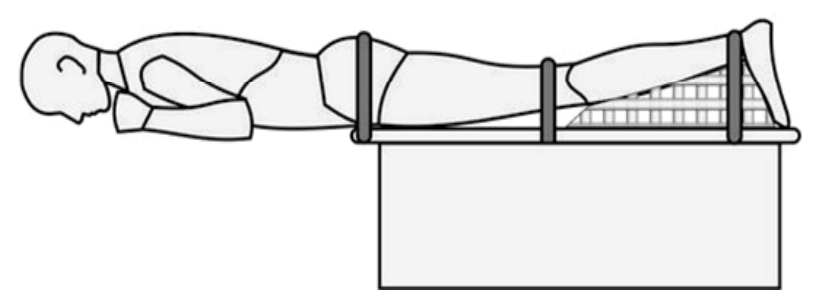

Figure 2.

Back extension test-(BET).

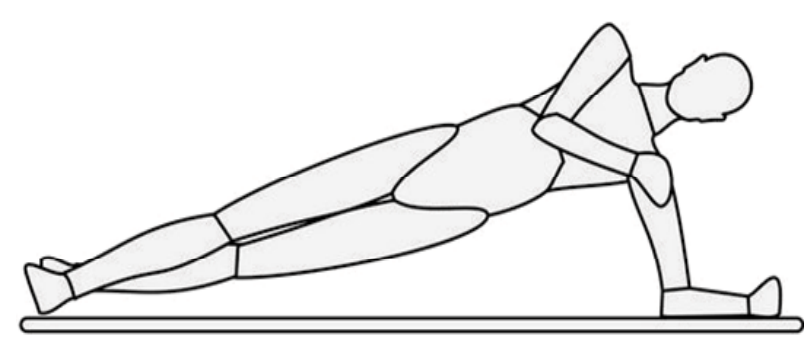

Figure 3.

Side bridge torso test-(RSB \& LSB).

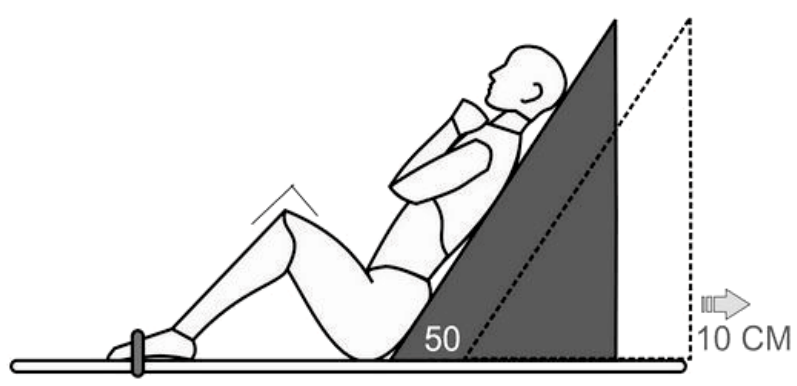

Figure 4.

Flexor endurance test-(FET).

as in the case of fatigue, and/or there were any signs of pain and/or when a maximum time of 300 seconds was reached. In all endurance tests subjects were encouraged to maintain their proper positions and straight posture as long as they could.

\section{Data Analyses}

Analysis of the effects of posture and torso muscular endurance was conducted with four (one per postural group) General Linear Model (GLM) univariate analyses. The GLMs were held to a significance level of alpha $=0.05$. However, during interpretation the alpha scores were held to adjusted standard of one fourth the original to account for the multiple comparisons. In order to assess which of the postures were associated with the differences in endurance scores pairwise comparisons were evaluated against the protected $\mathrm{F}$ values in the omnibus test. All statistical analysis were conducted in SPSS software portfolio (IBM SPSS v19).

\section{Results}

Posture assessments were able to identify four high prevalence postures in each group within this childhood population (Table 1). Primary analysis for the effect of posture on endurance revealed a statistically significant effect for the BET (F = $5.344, p<0.01)$. There were no other statistically significant differences based on our adjusted requirement $(p<0.02)$ for multiple comparisons. To assess the postural condition that caused the main effect pairwise comparisons were performed, they demonstrated that those with lordotic spine postures were less resilient (in extension endurance) than those with good, kyphotic and scoliotic postures $(p<0.01 ; p<0.01 ; p<0.01$; respectively). The measure of mean endurance scores and standard deviations (SD) stratified by postural subgroup are presented in Table 2.

Secondary analysis of this data set was done to inform and direct further research into adolescent posture and endurance scores. Upon examination and for descriptive purposes only; males seemed to be more likely to have hyper-kyphotic postures while females were more likely to have hyper-lordotic postures. Counts and percentage scores for postural group analysis can be found in Table 1. Examining the effect of these potential differences and how gender and age may play a factor is beyond the scope of this paper.

\section{Discussion}

This study provided information on the relationship between spine posture and torso endurance in school aged children from 
Table 2.

Mean (SD) scores for each isometric endurance test stratified by posture.

\begin{tabular}{ccccc}
\hline \multirow{2}{*}{ Posture } & \multicolumn{4}{c}{ Endurance Time (s) } \\
\cline { 2 - 5 } & BET $^{*}$ & FET & LSB & RSB \\
& $(\mathrm{F}=5.344 ; p<0.01)$ & $(\mathrm{F}=2.670 ; p<0.05)$ & $(\mathrm{F}=3.050 ; p=0.03)$ & $(\mathrm{F}=1.967 ; p=0.12)$ \\
\hline Good & $176.09(68.38)$ & $134.09(77.75)$ & $77.15(42.37)$ & $82.39(43.17)$ \\
Lordotic & $155.34(68.74)^{* *}$ & $122.01(77.84)$ & $70.64(34.79)$ & $72.57(35.66)$ \\
Kyphotic & $176.82(71.18)$ & $143.13(84.99)$ & $78.62(35.38)$ & $81.41(36.47)$ \\
Scoliotic & $178.08(67.73)$ & $133.47(75.15)$ & $76.03(36.53)$ & $80.73(36.66)$ \\
\hline
\end{tabular}

Note: ${ }^{*}$ Statistical significance for omnibus test held at $p<0.0125$ to adjust for multiple comparisons; ${ }^{* *}$ Statistical significance for each Pairwise comparisons held at $p<0.05$.

7 to 14 years of age. Each of the postural clusters were well represented in the sample of school aged children, with slightly more lordotic and kyphotic postures followed by scoliotic and good postured children (Table 1). Our hypothesis was supported by the data set, in that posture did affect torso isometric muscle endurance. The results show that there was a statistically significant decrease in back extensor endurance in those with lordotic posture as shown by the BET test (Table 2). Moreover, if not for adjusting the $\mathrm{p}$ values for multiple comparisons, the data also showed trends for lordosis decreasing both RSB and LSB endurance scores. Those with kyphotic posture showed trends towards increased anterior chain torso endurance as indicated by the FET (Table 2).

To our knowledge, this is the first study to establish a relationship between spine posture and isometric torso muscle endurance profiles in children aged 7 to 14 years. Regardless of the limited data for direct comparison to our results, there is a body of similar work that may provide further insights when considering the ramifications of posture and endurance in children. Mulhearn and George (1999) have documented that those gymnasts with lordotic posture had poorer exercise execution compared with ideal (normal) or sway-back (kyphotic) posture. It was speculated that increased lumbar curvature may have affected these gymnasts, which is consistent with our data set. Supporting evidence from Coorevits et al., (2005) revealed that lumbar curvature could be a possible factor to influence back muscle endurance assessment, which was carefully considered here. Lastly, the postural analysis in our studied showed that boys are more prone toward kyphotic posture and girls to lordosis (Table 1). These findings were congruent with Mulhearn and George's (1999) data set of gymnasts. However, this was in contrary to Awad \& Atta-Allah (2012) who documented kyphosis is more common in girls.

Raistenskis et al. (2012) evaluated 103 children aged between 7 to 17 and recorded significantly lower torso muscle endurance values compared with our data. These authors concluded that increased torso endurance has a positive influence on posture, which is inconsistent with our results. A study by Bharati and Rati (2012) showed that with an increase in postural thoracic kyphosis, there is no significant difference in the strength and endurance of the rectus abdominis, internal and external obliques and transverse abdominis muscles in subjects with and without kyphosis aged from 20 to 50. Our study showed similar results, though trends suggested that there may be some gains in anterior chain endurance. Sinaki and associates (1996) found that those with stronger back extensors have smaller thoracic kyphosis and larger lumbar lordosis with increased sacral inclination, at least in adult women. In contrast to this, we have observed that increased lumbar lordosis resulted in lower torso extension endurance compared to the other postural groups, as shown in Table $2(p<0.05)$.

A number of anatomical and biomechanical factors may be considered when examining the relationship between form and function. Interesting findings were documented in Anwajler and associates (2006) study; they found that force-velocity parameters are balanced within thoracic kyphosis and lumbar lordosis groups. It remains unclear the nature of the relationship between gross anatomical posture and muscular microanatomy, such as optimal muscle rest length conditions. These questions will likely need to be addressed to fully understand the relationships between maturation, posture and muscular endurance, and possible risk factors for conditions such as low back pain.

Our study has certain limitations, only associations can be drawn from this data set, it remains unclear if posture causes changes in endurance, or vice versa. Also, analyses of spinal postures were obtained using a grading system rather than a more direct measure of spinal deviation such as radiographic measures in degrees. However, for large samples such as this, exposure of ionizing radiation is likely unnecessary. The technique used here is also readily available and accessible in most clinical situations or even in coaching/teaching instances. Finally, the subjects were not separated into athletic and non-athletic groups and parallel testing was conducted. It is possible that, by way of competition, some motivation factors were affected. However, due to the nature of the testing and lack of material incentives these affects would be negligible. It is also possible that certain athletic groups may favour certain spine postures but this was not considered in this study. The upper limit of 300 seconds for each endurance test was set as such, as this was found to be the limit for which subjects could maintain correct form without the use of compensation strategies and additional musculature (i.e. cervical extension, hamstring activation etc.). Also, safety concerns such as fatigue induced pain, over exertion and intensive breathing would become apparent beyond 300 seconds. Despite this being a limitation, letting subjects go beyond 300 seconds would only further enhance our statistical significance. The kyphotic group was comprised of more males and older subjects, as was the lordotic group composed of more females and younger subjects. This age and gender relation to spine posture may influence the endurance times, however this is the natural distribution of subjects within these groups and the real link among these factors is unknown. 


\section{Conclusion}

This first attempt to examine spine posture and endurance scores will help interpret pain mechanisms and spine function in the future. In addition this database may give context for interpreting tests results from children for both therapeutic and performance training goals.

\section{Acknowledgements}

The financial support of the Natural Sciences and Engineering Research Council, Canada is gratefully acknowledged. The authors wish to thank the valuable contributions made by the Dean, the parents and all the children from the elementary school for their help, support and participation in this study.

\section{REFERENCES}

Anwajler, J., Skrzek, A., Mraz, M., Skolimowski, T., \& Wozniewski, M. (2006). The size of physiological spinal curvatures and functional parameters of trunk muscles in children with idiopathic scoliosis. Isokinetic and Exercise Science, 14, 251-259.

Bharati, A., \& Rati, G. P. (2012). A study to correlate postural thoracic kyphosis and abdominal muscle strength and endurance. Indian Journal of Physiotherapy and Occupational Therapy-An International Journal, 6, 19-21.

Breen, R., Pyper, S., Rusk, Y., \& Dockrell, S. (2007). An investigation of children's posture and discomfort during computer use. Ergonomics, 50, 1582-1592. doi:10.1080/00140130701584944

Coorevits, P. L. M., Danneels, L. A., Ramon, H., Van Audekercke, R., Cambier, D. C., \& Vanderstraeten, G. G. (2005). Statistical modeling of fatigue-related electromyographic median frequency characteristics of back and hip muscles during a standardized isometric back extension test. Journal of Electromyogr Kinesiology, 15, 444-451. doi:10.1016/j.jelekin.2005.02.002

Côté, P., Kreitz, B., Cassidy, J. D., Dzus, A. K., \& Martel, J. A. (1998). Study of the diagnostic accuracy and reliability of the scoliometer and Adam's forward bend test. Spine, 23, 796-802. doi:10.1097/00007632-199804010-00011

Dankaerts, W., O'Sullivan, P., Burnett, A., \& Straker, L. (2006). Differences in sitting postures are associated with nonspecific chronic low back pain disorders when patients are subclassified. Spine, 31, 698-704. doi:10.1097/01.brs.0000202532.76925.d2

Dejanović, A., Harvey, E., \& McGill, S. M. (2012). Changes in 3D torso muscle endurance profiles in children aged 7 to 14 : Reference values. Archives of Physical Medicine and Rehabilitation, 93, 22952301. doi:10.1016/j.apmr.2011.12.023

Escalada, F., Marco, E., Duarte, E., Muniesa, J. M., Belmonte, R., Tejero, M., \& Caceres, E. (2005). Growth and curve stabilization in girls with adolescent idiopathic scoliosis. Spine, 30, 411-417. doi:10.1097/01.brs.0000153397.81853.6a

Evans, K., Refshauge, K., \& Adams, R. (2007). Trunk muscle endurance tests: Reliability, and gender differences in athletes. Journal of Science and Medicine in Sport, 10, 447-455. doi:10.1016/j.jsams.2006.09.003

Griegel-Morris, P., Larson, K., Mueller-Klaus, K., \& Oatis, C. A. (1992). Incidence of common postural abnormalities in the cervical, shoulder, and thoracic regions and their association with pain in two age groups of healthy subjects. Physical Therapy, 72, 425-431.

Harrison, D. E., Cailliet, R., Harrison, D. D., \& Janik T. J. (2002) How do anterior/posterior translations of thoracic cage affect the sagittal lumbar spine, pelvic tilt and thoracic kyphosis? European Spine Journal, 11, 287-293. doi:10.1007/s00586-001-0350-1

Harrison, D. D., Harrison, S. O., Croft, A. C., Harrison, D. E., \& Troyanovich, S. J. (1999). Sitting biomechanics part I: Review of the literature. Journal of Manipulative and Physiological Therapeutics, 22, 594-609. doi:10.1016/S0161-4754(99)70020-5

Hrysomallis, C., \& Goodman, C. (2001). A review of resistance exercise and posture realignment. Journal of Strength and Conditioning Research, 15, 385-390.

Kendall, E. P., McCreary, E. K., Provance, P. G., Rodgers, M. Mc., \& Romani, W. A. (2005). Muscles testing \& function with posture and pain (5th ed.). Baltimore: Williams \& Wilkins.

Malina, R. M, Bouchard, C., \& Bar-Or, O. (2004). Growth, maturation, and physical activity (2nd ed.). Champaign, IL: Human Kinetics.

McEvoy, M. P., \& Grimmer, K. (2005). Reliability of upright posture measurements in primary school children. BMC Musculoskeletal Disorders, 6, 35. doi:10.1186/1471-2474-6-35

McGill, S. M. (2007). Low back disorders: Evidence based prevention and rehabilitation (2nd ed.). Champaign, IL: Human Kinetics.

McGill, S. M., Childs, A., \& Liebenson, C. (1999). Endurance times for low back stabilization exercises: Clinical targets for testing and training from a normal database. Archives of Physical Medicine and Rehabilitation, 80, 941-944. doi:10.1016/S0003-9993(99)90087-4

Mulhearn, S., \& George, K. (1999). Abdominal muscle endurance and its association with posture and low back pain. Physiotherapy, 85, 210-216. doi:10.1016/S0031-9406(05)65666-0

Raistenskis, J., Sinkevičius, R., Varniene, L., \& Doveikiene, J. (2012). Association between back pain and physical development in children. Health Sciences Magazine, 22, 11-16.

Ramirez, N., Johnston, C. E., \& Browne, R. H. (1997). The prevalence of back pain in children who have idiopathic scoliosis. Journal of Bone Joint Surgery of America, 79, 364-368.

Reamy, B. V., \& Slakey, J. B. (2001). Adolescent idiopathic scoliosis: Review and current concepts. American Family Physician, 64, 111117.

Sanders, J. O., Browne, R. H., Cooney, T. E. Finegold, D. N., McConnell, S. J., \& Margraf, S. A. (2006). Correlates of the peak height velocity in girls with idiopathic scoliosis. Spine, 31, 2289-2295. doi:10.1097/01.brs.0000236844.41595.26

Sinaki, M., Itoi, E., Rogers., J. W., Bergstralh, E. J., \& Wahner, H. W. (1996). Correlation of back extensor strength with thoracic kyphosis and lumbar lordosis in estrogen-deficient women. American Journal of Physical Medicine \& Rehabilitation, 75, 370-374. doi:10.1097/00002060-199609000-00013

Sparto, P. J., Parnianpour, M., Reinsel, T. E., \& Simon, S. (1997). The effect of fatigue on multijoint kinematics and load sharing during a repetitive lifting test. Spine, 22, 2647-2654. doi:10.1097/00007632-199711150-00013

Stokes, I. A. F., \& Gardner-Morse, M. (2004). Muscle activation strategies and symmetry of spinal loading in the lumbar spine with scoliosis. Spine, 29, 2103-2107. doi:10.1097/01.brs.0000141182.42544.1f

Toppenberg, K., \& Bullock, M. (1986). The interrelation of spinal curves, pelvic tilt and muscle length in the adolescent female. Australian Journal of Physiotherapy, 32, 6-12.

Weinstein, S. (1994). Adolescent idiopathic scoliosis: Prevalence and natural history. The Pediatric Spine: Principles and Practic, 463-478.

Wilke, H. J., Neef, P., Caimi, M., Hoogland, T., \& Claes L. E. (1999). New in vivo measurements of pressures in the intervertebral disc in daily life. Spine, 24, 755-762.

doi:10.1097/00007632-199904150-00005 